\title{
Active Disturbance Rejection Control with Low Power Extended State Observer and its Application to Speed Control of Single Shaft Gas Turbines
}

\author{
Hao Xia, Xiaoyang Li
}

Abstract - We propose a low power active disturbance rejection control (LPADRC) with outstanding high frequency noise attenuation and low power (or low exponent of the gain) structure. We prove the performance recovery property of the proposed scheme with perturbation theory. By performance recovery, we mean that the performance could be sufficiently close to the performance of the nominal system. Moreover, Bode-magnitude plot was used to demonstrate the better high-frequency noise attenuation compared to linear active disturbance rejection control (LADRC). We apply this new controller to speed control of gas turbines, which usually suffers from unknown model uncertainties and external disturbances such as changes in ambient temperature, pressure, and electric load. Other handicaps include high-frequency measurement noise from the angular velocity sensor. The effectiveness of the proposed gas turbine control scheme is verified with a highfidelity simulation model.

Index Terms - Low power extended state observer, Active disturbance rejection control, Performance recovery, High frequency measurement noise, Single shaft gas turbine

\section{INTRODUCTION}

In contrast to model-based control methods, active disturbance rejection control (ADRC), due to its low dependence on model accuracy, satisfactory disturbance rejection performance and simple tuning procedure [1], has developed rapidly in recent years and been applied to a wide range of industrial applications (e.g., [2] [3] [4]).

Gas turbines have been widely used as prime movers in power systems due to its large ratio of power-volume and fast start-up [5]. Among all types of gas turbines, the single shaft gas turbine (SSGT) is most suited for power plant field [6]. During its operation, SSGT often suffers from external disturbance such as changes in electric load and ambient temperature. Furthermore, due to the use of rotational speed sensor, there always exists high-frequency measurement noise [7]. To endow the system with a satisfactory operation, the industry needs a powerful and easy to be tuned controller.

This work is supported by National Natural Science Foundation of China $(61973052,61273098)$ and National Science and Technology Major Project2017-V-0005-0055.

Hao Xia, is with the Electrical Engineering Department, Dalian University of Technology, Dalian, Liaoning 116024, China (e-mail: Hao.X@dlut.edu.cn).

Xiaoyang Li is with the Electrical Engineering Department, Dalian University of Technology, Dalian, Liaoning 116024, China (e-mail: Lixiaoyang@mail.dlut.edu.cn).
For model-based control methods, modeling the plant is the first step. However, for complex machines with high non-linearity and model-uncertainty like gas turbines, it would be too complicated to model them accurately [8]. But for ADRC, both model-uncertainty and external disturbance are considered as the matched "total disturbance", and their effects could be estimated and then canceled via some appropriate feedback laws [1] [9]. If the disturbance were canceled completely, the system would be transformed into a multi-integrator series, which we will call it the "nominal model". It might sounds too ideal, but at least we can make the performance of the system infinitely close to that of the nominal system, under certain conditions; that is called "performance recovery". So far, only LADRC has been rigorously proved to have that property in [10] (not the same name but identical structure); other variants of ADRC lack or have not been proved to have it. This superiority and the simple tuning procedure together make LADRC widely deployed in the past few years. However, there exist three problems for applying LADRC directly to the SSGT control: 1) A trade off between the fast convergence rate and the high sensitivity to high-frequency measurement noise. When the measurement output is contaminated severely by high-frequency noise, the bandwidth (or gain) of linear extended state observer (LESO), which is the core component of LADRC, is severely limited. 2) The value of the observer parameter $w_{o}^{n+1}$ is too large for some digital implementations. 3) There are unmatched total disturbances in the original nonlinear model of SSGT.

To solve the first and second two problems, we replace the LESO with low power extended state observer (LPESO), which was proposed separately in [11] and [12]. The LPESO, with parameters no larger than $w_{o}^{2}\left(w_{o} \gg 1\right)$, possesses outstanding high-frequency measurement noise attenuation while preserves the same exponential errordecay speed of LESO [12]. In the current paper, we will study it further by analyzing the performance recovery of it via perturbation theory.

More interestingly, we found a simple and fast parameter configuration method (compared to the comprehensive but rather complex procedure proposed in [13]) for any $2 n$-th order high gain observers with this "low power" structure; this new method places all poles of observer at -1 , because it is usually the value of gain that matters, 
rather than value of other parameters [14]. Based on this observer parameter configuration method, we proposed a useful parameter-tuning procedure for LPADRC with the same number of parameters to be tuned, which is the same as LADRC; that makes LPADRC distinguished with other strategies such as LADRC with a low-pass filter [15] and other nonlinear ADRC [16]. These features together make LPADRC an appealing choice for the speed control of SSGT.

Before designing LPADRC according to the order of the original SSGT model, we first decompose the model into an internal and an external dynamic to obtain its relative degree, which solves the third problem aforementioned, i.e., the unmatched disturbance in the original SSGT model. Then a 2nd-order, instead of 3rd-order LPADRC, will be designed,

To summarize, the contributions of this paper are:

1) Low power active disturbance rejection control was proposed and the performance recovery was proved.

2) This new ADRC possesses two merits, i.e. better high-frequency noise attenuation and lower exponent of gain compared to LADRC.

3) A systematic parameter-tuning method was given, in conjunction with a easy to follow flow-chart.

4) By introducing the concept of relative degree, we bridged the gap between ADRC and speed control of single shaft gas turbines .

The rest of this paper are organized as follows. The design of LPADRC, performance recovery analysis and tuning procedure are given in Section II. The general nonlinear dynamic model of SSGT is given in Subsection III-A. A linearized model for a specific SSGT, 2nd-order LPADRC design, and a Bode-magnitude plot analyzing noise-sensitivity of both LPESO and LESO, are given in Subsection III-B. Numerical simulations using TMATS toolbox on MATLAB/Simulink are given in Subsection III-C to compare the performance of PI, LADRC and LPADRC. Conclusions are discussed in Section IV.

\section{LOW POWER EXTENDED STATE OBSERVER BASED ADRC}

\section{A. Notations}

The notation $\mathbb{R}$ stands for the set of real numbers and $\mathbb{D}$, $\Omega$ denote compact sets. States $\mathbf{x}(t), \mathbf{w}(t), \xi(t), \zeta(t), \mathbf{z}(t)$ are functions of time $t$; so do output $y(t), y^{*}(t)$, which represent the output of the actual system and the ideal linear system, respectively. For the sake of convenience, we will write them in the form of $\mathbf{x}, \mathbf{w}, \xi, \zeta, \mathbf{z}, y, y^{*}$. Capital letter $V$, with subscripts $0,1,2,3,^{\prime}$ are taken to denote Lypunov function

\section{B. Observer and controller design}

Given the $n$ th-order nonlinear SISO system (1).

$$
\begin{aligned}
\dot{\mathbf{x}} & =f(\mathbf{x}, \mathbf{w})+g(\mathbf{x}, \mathbf{w}) u \\
y & =h(\mathbf{x})
\end{aligned}
$$

where $\mathbf{x} \in \mathbb{R}^{n}, u \in \mathbb{R}, y \in \mathbb{R}, \mathbf{w} \in \mathbb{D}_{\mathbf{w}} \subset \mathbb{R}^{m}$ where $\mathbb{D}_{\mathbf{w}}$ is a compact set. Functions $f(\mathbf{x}, \mathbf{w}), g(\mathbf{x}, \mathbf{w}), h(\mathbf{x})$ are continuously differentiable in $(\mathbf{x}, \mathbf{w})$ over $\mathbb{R}^{n} \times \mathbb{D}_{\mathbf{w}}$. Moreover, $f(0,0)=0, g(0,0)=0$ and $\mathbf{w}$ is continuously differentiable in $t$ on $\mathbb{D}_{\mathbf{w}}$.

Let the $p-t h$ derivative of $y(t)$ along $\mathbf{x}(t)$ (the solution of equation (1)) be represented by:

$$
y^{(p)}(t)=\frac{d^{p} y}{d t^{p}}(t)=f_{\text {part }, p}(\mathbf{x}, \mathbf{w})+g_{\text {part }, p}(\mathbf{x}, \mathbf{w}) u
$$

where $f_{\text {part }, p}$ and $g_{\text {part, } p}$ are continuously differentiable functions defined on $\mathbb{R}^{n} \times \mathbb{D}_{\mathbf{w}}$.

Definition 1: [17] The nonlinear system (1) is said to have relative degree $\varrho \in(0, n]$ if $g_{\text {part }, i}(\mathbf{x}, \mathbf{w})=0$ for $i=1, \cdots, \varrho-2$ and $g_{\text {part }, \varrho-1} \neq 0$.

Lemma 1: [18] If system (1) has relative degree $\rho<n$, there exists a diffeomorphism $\mathcal{T}(\mathbf{x})$ over $\mathbb{R}^{n}$ such that

$$
\overline{\mathbf{x}}=\mathcal{T}(\mathbf{x}) \stackrel{\text { def }}{=}\left(\begin{array}{c}
\phi(\mathbf{x}) \\
--- \\
\psi(\mathbf{x})
\end{array}\right) \stackrel{\text { def }}{=}\left(\begin{array}{c}
\zeta \\
--- \\
\xi
\end{array}\right)
$$

By this transformation, the system would be:

$$
\begin{aligned}
& \dot{\zeta}=f_{0}(\zeta, \xi, \mathbf{w}) \\
& \dot{\xi}_{i}=\xi_{i+1}, \quad i=1, \cdots, \varrho-1 \\
& \dot{\xi}_{\varrho}=\phi_{1}(\zeta, \xi, \mathbf{w})+\phi_{2}(\zeta, \xi, \mathbf{w}) u
\end{aligned}
$$

where $\zeta \in \mathbb{R}^{n-\varrho}, \xi=\left(\xi_{1}, \cdots, \xi_{\varrho}\right) \in \mathbb{R}^{\varrho}$. Vector $\mathbf{w}$ is the external disturbance. Unknown functions $f_{0}(\cdot), \phi_{1}(\cdot), \phi_{2}(\cdot)$ are continuously differentiable in $(\zeta, \xi, \mathbf{w})$ over $\mathbb{R}_{n-\varrho} \times$ $\mathbb{R}_{\varrho} \times \mathbb{D}_{\mathbf{w}}$ with $\phi_{2}(\cdot)$ satisfying $\phi_{2}(\cdot)>$ constant $_{1}>0$ where constant $_{1}$ is a positive constant.

In this way we obtain what is known as the normal form of the system with the internal dynamic $\zeta$ and external dynamic $\xi$. More importantly, in the external dynamic, the total disturbance fulfills the requirement of "matched" [9], which requires that the total disturbance and the control signal are in the same channel. Definition 1 will latter be used to obtain the relative degree of nonlinear dynamic model of single shaft gas turbine.

We impose a restriction on the system being minimumphase. Hence, we need make some assumptions about the internal dynamic $\zeta$. For linear system, Assumption 1 indicates that there is no zeros with a positive real part.

Assumption 1: There exists a radially unbounded positive definite function $V_{0}(\zeta)$ such that for all $\zeta \in \mathbb{R}^{n-\varrho}$, $\xi \in \mathbb{R}^{\varrho}$ and $\mathbf{w} \in \mathbb{D}_{\mathbf{w}}, V_{0}$ satisfies

$$
\frac{\partial V_{0}}{\partial \zeta} f_{0}((\zeta, \xi, \mathbf{w}) \leq 0, \quad \forall\|\zeta\| \geq \chi(\xi, \mathbf{w})
$$

where $\chi(\cdot)$ is a nonnegative continuous function.

According to Assumption 1, by Lyapunov converse theorem(see [18]), there exists a non-decrease nonnegative function $\alpha(\cdot)$ satisfying $\lim _{x \rightarrow \infty} \alpha(x)=\infty$ and a nonnegative constant $\mu_{0}$ such that for any positive $\mu$, $\Omega_{\zeta}=\left\{V_{0}(\zeta) \leq \mu_{0}+\alpha(\mu)\right\}$ is a positively invariant set for all $\xi \in \Omega_{\xi}=\left\{V^{\prime}(\xi) \leq \mu\right\}$ where $V^{\prime}(\xi)=\xi^{T} \mathcal{P} \xi$; matrix $\mathcal{P}$ is the positive solution of Lyapunov equation

$$
\mathcal{P}\left(A-B K_{c}\right)+\left(A-B K_{c}\right)^{T} \mathcal{P}=-I
$$


where

$$
K_{c}=\left[\begin{array}{llll}
\beta_{1} w_{c}^{\varrho} & \beta_{2} w_{c}^{\varrho-1} & \cdots & \beta_{\varrho} w_{c}
\end{array}\right]_{1 \times \varrho}
$$

with $w_{c}>0$ and $\beta_{i}, 1 \leq i \leq \varrho$ are chosen such that

$$
s^{\varrho}+\beta_{\varrho} s^{\varrho-1}+\cdots+\beta_{1}
$$

is a Hurwitz polynomial.

Now define a new state $\xi_{\varrho+1}$ with a constant $b_{0}>0$

$$
\xi_{\varrho+1} \stackrel{\text { def }}{=} \phi_{1}(\zeta, \xi, \mathbf{w})+\left(\phi_{2}(\zeta, \xi, \mathbf{w})-b_{0}\right) u
$$

With the new extended state $\xi_{\varrho+1}$, we can transform system(4) into (6)

$$
\begin{aligned}
& \dot{\zeta}=f_{0}(\zeta, \xi, \mathbf{w}) \\
& \dot{\xi}=A \xi+B b_{0} u+B \xi_{\varrho+1}
\end{aligned}
$$

where

$$
A=\left[\begin{array}{ccccc}
0 & 1 & 0 & \cdots & 0 \\
0 & 0 & 1 & \ddots & 0 \\
\vdots & \ddots & 0 & 1 & \vdots \\
0 & \ddots & \ddots & 0 & 1 \\
0 & 0 & \cdots & 0 & 0
\end{array}\right]_{\varrho \times \varrho}, \quad B=\left[\begin{array}{c}
0 \\
0 \\
\vdots \\
0 \\
1
\end{array}\right]_{\varrho \times 1}
$$

Then, a low power extended state observer is given as

$$
\begin{array}{llll}
\dot{\hat{\xi}}_{i, 1} & =\hat{\xi}_{i, 2} & +k_{i, 1} w_{o} e_{i}, & i=1, \cdots, \varrho-2 \\
\dot{\hat{\xi}}_{i, 2} & =\hat{\xi}_{i+1,2} & +k_{i, 2} w_{o}^{2} e_{i}, & i=1, \cdots, \varrho-2 \\
\dot{\hat{\xi}}_{\varrho-1,1} & =\hat{\xi}_{\varrho-1,2} & +k_{\varrho-1,1} w_{o} e_{\varrho-1} & \\
\dot{\hat{\xi}}_{\varrho-1,2} & =\hat{\xi}_{\varrho, 2} & +k_{\varrho-1,2} w_{o}^{2} e_{\varrho-1} & +b_{0} u \\
\dot{\hat{\xi}}_{\varrho, 1} & =\hat{\xi}_{\varrho, 2} & +k_{\varrho, 1} w_{o} e_{\varrho} & +b_{0} u \\
\dot{\hat{\xi}}_{\varrho, 2} & = & k_{\varrho, 2} w_{o}^{2} e_{\varrho} &
\end{array}
$$

where $e_{1}=y-\hat{\xi}_{1,1}$ and $e_{i}=\hat{\xi}_{i-1,2}-\hat{\xi}_{i, 1}, i=2,3, \ldots, \varrho$ and $K_{i}=\left(k_{i, 1}, k_{i, 2}\right)^{T}, i=1,2, \ldots, \varrho$ are observer parameters. Extract $\varrho+1$ states as the approximation of $\xi, \xi_{\varrho+1}$ by

$$
\bar{\xi}=\mathcal{L} \hat{\xi}
$$

where $\hat{\xi}=\left(\hat{\xi}_{1,1}, \hat{\xi}_{1,2}, \cdots, \hat{\xi}_{2 \varrho, 1}, \hat{\xi}_{2 \varrho, 2}\right)^{T} \in R^{2 \varrho}$ and $\mathcal{L}$ is a block diagonal matrix defined as

$$
\mathcal{L}=\operatorname{diag}\left(\left[\begin{array}{ll}
1 & 0
\end{array}\right], \cdots,\left[\begin{array}{ll}
1 & 0
\end{array}\right]\right)_{\varrho \times 2 \varrho}
$$

The estimate of "total disturbance" can be canceled by choosing the feedback control law as

$$
\Gamma\left(\bar{\xi}, \hat{\xi}_{\varrho, 2}\right)=\frac{v-\hat{\xi}_{\varrho, 2}}{b_{0}}
$$

where $v$ is the feedback law which could be LQR, PD, or slide mode control.

In this paper we choose the feedback law to be:

$$
\Gamma\left(\bar{\xi}, \hat{\xi}_{\varrho, 2}\right)=\frac{-\left[\begin{array}{ll}
K_{c} & 1
\end{array}\right]\left[\begin{array}{c}
\bar{\xi} \\
\hat{\xi}_{\varrho, 2}
\end{array}\right]}{b_{0}}
$$

If the estimation error satisfies (10)

$$
\left\|\left[\begin{array}{c}
\xi \\
\xi_{\varrho+1}
\end{array}\right]-\left[\begin{array}{c}
\bar{\xi} \\
\hat{\xi}_{\varrho, 2}
\end{array}\right]\right\|=0
$$

for all $t \geq 0$ with $\|\cdot\|$ denoting the 2-norm, then substituting (9) into (6) leads to the target/nominal system:

$$
\begin{aligned}
& \dot{\zeta}=f_{0}(\zeta, \xi, \mathbf{w}) \\
& \dot{\xi}=\left(A-B K_{c}\right) \xi \\
& y=\xi_{1}
\end{aligned}
$$

In the following, we will consider the output of (11) as the "target output" which is decided by $K_{C}$ and denoted by $y^{*}(t)$.

In practice, due to the exist of peaking-phenomenon of LPESO [12], we need an analogous-saturation function, which was introduced in [10], to avoid needless large control signal by

$$
u=M g_{w_{o}}\left(\frac{\Gamma(\bar{\xi})}{M}\right)
$$

where

$$
g(x)=\left\{\begin{array}{lc}
x & 0 \leq|x| \leq 1 \\
\operatorname{sgn}(x)\left[|x|+w_{o}(|x|-1)-\frac{w_{o}\left(x^{2}-1\right)}{2}\right] & 1 \leq|x| \leq 1+w_{o}^{-1} \\
\operatorname{sgn}(x)\left(1+\frac{1}{2 w_{o}}\right) & 1+w_{o}^{-1} \leq|x|
\end{array}\right.
$$

Constant $M$ could be any positive constant satisfying

$$
M>\max _{(\zeta, \xi, \mathbf{w}) \in \Omega_{\zeta} \times \Omega_{\xi} \times \mathbb{D}_{\mathbf{w}}}\left|\frac{-\phi_{1}(\zeta, \xi, \mathbf{w})-K_{c} \xi}{\phi_{2}(\zeta, \xi, \mathbf{w})}\right|
$$

Generally, when the estimation error is small enough, the feedback control will satisfy

$$
\left|\Gamma\left(\bar{\xi}, \hat{\xi}_{\varrho, 2}\right)\right| \leq M
$$

The normal saturation function sat() has the similar function as $g(\cdot)$ while it would lead to a more complicated stability proof [10].

For the ease of analysis, we take the estimation error as

$$
\begin{aligned}
& \tilde{\xi}_{i}=\left(\xi_{i}, \xi_{i+1}\right)^{T}-\hat{\xi}_{i}, \quad \forall i=1, \cdots, \varrho-1 \\
& \tilde{\xi}_{\varrho, 1}=\xi_{\varrho}-\hat{\xi}_{\varrho, 1}
\end{aligned}
$$

$$
\tilde{\xi}_{\varrho, 2}=\phi_{1}(\cdot)+\left(\phi_{2}(\cdot)-b_{0}\right) M g_{w_{o}}\left(\frac{\Gamma\left(\xi, \hat{\xi}_{\varrho, 2}\right)}{M}\right)-\hat{\xi}_{\varrho, 2}
$$

and scale it by

$$
z_{i}=w_{o}^{2-i} D_{2}^{-1} \tilde{\xi}_{i}
$$

This scaling leads to a new representation given as

$$
\dot{\mathbf{z}}=w_{o} J z+B_{2 \varrho} w_{o}^{-\varrho} \Phi(t, \zeta, \xi, \mathbf{w})
$$

where

$$
J=\left[\begin{array}{ccccc}
E_{1} & N & 0 & \cdots & 0 \\
Q_{2} & E_{2} & N & \ddots & \vdots \\
0 & Q_{i} & E_{i} & N & 0 \\
\vdots & \ddots & \ddots & \ddots & N \\
0 & \cdots & 0 & Q_{\varrho} & E_{\varrho}
\end{array}\right], B_{2 \varrho}=\left(\begin{array}{c}
0 \\
\vdots \\
0 \\
1
\end{array}\right)
$$


and $E_{i}, Q_{i}$, and $N$ are matrices defined as

$$
E_{i}=A_{2}-K_{i} C_{2}, \quad Q_{i}=\left[\begin{array}{ll}
0 & k_{i, 1} \\
0 & k_{i, 2}
\end{array}\right], N=\left[\begin{array}{ll}
0 & 0 \\
0 & 1
\end{array}\right]
$$

with

$$
A_{2}=\left[\begin{array}{ll}
0 & 1 \\
0 & 0
\end{array}\right] \quad B_{2}=\left(\begin{array}{l}
0 \\
1
\end{array}\right) C_{2}=\left(\begin{array}{ll}
1 & 0
\end{array}\right) D_{2}=\left[\begin{array}{cc}
w_{o} & 0 \\
0 & w_{o}^{2}
\end{array}\right]
$$

and $\Phi(\cdot)$ is a scalar function which will be defined explicitly in Section II-C.

\section{Performance recovery}

In this subsection, we are to show that, if $M$ is chosen appropriately and $w_{o}$ is large enough, then the output $y$ of the whole system composed of $(4)(7)(12)$, could be infinitely close to the output $y^{*}$ of the target system (11).

Theorem 1: For closed-loop system consisting of the plant (4), LPESO (7), and feedback control law (12), if $b_{0}$ is chosen such that

$$
\max _{(\zeta, \xi, \mathbf{w}) \in \Omega_{\zeta} \times \Omega_{\xi} \times \mathbb{D}_{\mathbf{w}}}\left|\frac{\phi_{2}(\zeta, \xi, \mathbf{w})-b_{0}}{b_{0}}\right| \leq \frac{1}{\|G(s)\|_{\infty}}
$$

where

$$
\begin{aligned}
& G\left(\frac{s}{w_{o}}\right)=C^{1}\left(\frac{s}{w_{o}} I-J\right)^{-1} B \\
& \|G(s)\|_{\infty}=\left\|G\left(\frac{s}{w_{o}}\right)\right\|_{\infty}=\max _{\omega \in R}|G(j \omega)| \\
& C^{1}=\left(\begin{array}{lllllll}
1 & 0 & \cdots & 0 & 1 & -1 & 0
\end{array}\right)
\end{aligned}
$$

and $J$ is a Hurwitz matrix, then there exists $w_{o}^{*}>0$ such that for every $w_{o}>w_{o}^{*}$ :

- $\lim _{w_{o} \rightarrow \infty}\left|y(t)-y^{*}(t)\right|=0, \forall t>0$

- $|y(t)| \leq O\left(w_{o}^{-1}\right), \forall t>T_{1}\left(w_{c}\right)>0$

where $y^{*}(t)$ is the "target output" of system (11), $T_{1}\left(w_{c}\right)$ is a non-increasing function of $w_{c}$, and $O\left(w_{o}^{-1}\right)$ satisfies

$$
\lim _{w_{o} \rightarrow \infty} O\left(w_{o}^{-1}\right)=0
$$

Proof: The proof is along the spirit of [10] but with modifications. It generally includes two steps. First, prove that the estimation-error will enter a neighborhood of the origin within finite time. Both the period of time and radius of the neighborhood depend on the gain $w_{o}$. Then, show that $\xi$ also will decay into a neighborhood of the origin. More importantly, they will never leave these neighborhoods.

Now, we represent the closed-loop system by

$$
\begin{aligned}
& \dot{\zeta}=f_{0}(\zeta, \xi, \mathbf{w}) \\
& \dot{\xi}=A \xi+B\left(\phi_{1}(\cdot)+\phi_{2}(\cdot) u\right) \\
& \dot{\mathbf{z}}=w_{o} J \mathbf{z}+B_{2 \varrho} w_{o}^{-\varrho} \Phi(t, \zeta, \xi, \mathbf{w})
\end{aligned}
$$

where

$$
\begin{aligned}
& \Phi=k_{\varrho, 2} w_{o}^{\varrho+1}\left(z_{\varrho-1,2}-z_{\varrho, 1}\right)\left(-1+\frac{\left(\phi_{2}(\cdot)-b_{0}\right)}{b_{0}} g_{w_{o}}^{\prime}(\cdot)\right) \\
& +\left[\dot{\phi}_{1}+\dot{\phi}_{2} M g_{w_{o}}\left(\frac{\Gamma\left(\xi, \hat{\xi}_{\varrho, 2}\right)}{M}\right)+\frac{\left(\phi_{2}(\cdot)-b_{0}\right) g_{w_{o}}^{\prime}(\cdot)\left(-K_{C}\right) \dot{\xi}}{b_{0}}\right] \\
& \stackrel{\text { def }}{=} w_{o}^{\varrho+1} \Lambda_{1} B_{2 \varrho} C^{1} z+B_{2 \varrho}^{T} \Delta_{1}(\cdot)
\end{aligned}
$$

where

$$
\Lambda_{1}=\left(-1+\frac{\left(\phi_{2}(\cdot)-b_{0}\right)}{b_{0}} g_{w_{o}}^{\prime}(\cdot)\right)
$$

and $\Delta_{1}$ satisfies

$$
\begin{aligned}
\left|\Delta_{1}\right| \leq & \left\|\frac{\partial \phi_{1}}{\partial \xi} \dot{\xi}+\frac{\partial \phi_{1}}{\partial \zeta} \dot{\zeta}+\frac{\partial \phi_{1}}{\partial \mathbf{w}} \dot{\mathbf{w}}\right\|+\left\|\frac{\partial \phi_{2}}{\partial \xi} \dot{\xi}+\frac{\partial \phi_{1}}{\partial \zeta} \dot{\zeta}+\frac{\partial \phi_{2}}{\partial \mathbf{w}} \dot{\mathbf{w}}\right\| M \\
& +\left\|\frac{\left(\phi_{2}(\cdot)-b_{0}\right) g_{w_{o}}^{\prime}(\cdot)\left(-K_{c}\right) \dot{\xi}}{b_{0}}\right\| \\
\leq & c_{1}\|\xi\|+c_{2}\|\zeta\|+c_{3}
\end{aligned}
$$

for some positive constants $c_{i}>0, i=1,2,3$.

By the singular perturbation theory, we decompose the system into a fast dynamic $\mathbf{z}(t)$ and a slow dynamic $\zeta(t), \xi(t)$. We are to show that, the decay speed of $\|\mathbf{z}\|$, from $\mathbf{z}=\mathbf{z}(0)$ to sufficiently small, is faster than the dynamic of $\xi$, and, hence, as we stated before, the dynamic of system (18) would be very close to system (11).

Now, consider system (20)

$$
\dot{\mathbf{z}}=w_{o} J \mathbf{z}+w_{o} \Lambda_{1} B C^{1} \mathbf{z}
$$

According to circle criteria, the system (20) is exponentially stable, and by Kalman-Yakubovich-Popov (KYP) Lemma [18], there exists a Lyapunov function $V_{1}=z^{T} P_{z} z$ whose derivative along $z(z(0), t)$ (the solution of $(20))$ is bounded by $-w_{o} L_{0} V_{1}$ with $L_{0}>0$ a positive constant [14]. The derivative of $V_{1}$ along system (19) is derived:

$$
\begin{aligned}
\dot{V}_{1} & \leq-w_{o} L_{0} V_{1}+z^{T} P \Phi(t, \zeta, \xi, \mathbf{w}) \\
& \leq-w_{o} L_{0} \lambda_{1}\|\mathbf{z}\|^{2}+w_{o}^{-\varrho}\|\mathbf{z}\|\left\|P_{z}\right\|\left(c_{1}\|\xi\|+c_{2}\|\zeta\|+c_{3}\right) \\
& \leq-w_{o} L_{0} \lambda_{1}(1-\theta)\|\mathbf{z}\|^{2} \\
& -\|\mathbf{z}\|\left\|P_{z}\right\|\left[w_{o} \theta L_{0} \lambda_{1}\|\mathbf{z}\|-w_{o}^{-\varrho}\left(c_{1}\|\xi\|+c_{2}\|\zeta\|+c_{3}\right)\right] \\
& \leq-w_{o} L_{0}(1-\theta)\|\mathbf{z}\|^{2}, \forall\|\mathbf{z}\| \geq \frac{\left\|P_{z}\right\|\left(c_{1}\|\xi\|+c_{2}\|\zeta\|+c_{3}\right)}{w_{o}^{\varrho+1} \theta L_{0} \lambda_{1}}
\end{aligned}
$$

for some positive constant $\theta \in(0,1)$ and $\lambda_{1}=\lambda_{\min }\left(P_{z}\right)$. There exists $L_{1}>0$ such that

$$
\left\{\|\mathbf{z}\| \leq \frac{c_{1}\|\xi\|+c_{2}\|\zeta\|+c_{3}}{w_{o}^{\varrho+1} \theta L_{0} \lambda_{1}}\right\} \subset \Omega_{\mathbf{z}}
$$

where

$$
\Omega_{\mathbf{z}}=\left\{\mathbf{z} \in \mathbb{R}^{2 \varrho} \mid\|\mathbf{z}\| \leq L_{1} w_{o}^{-(\varrho+1)}\right\}
$$

By comparison lemma [18] and the property of quadratic function $\lambda_{\min }\left(P_{z}\right)\|\mathbf{z}\| \leq V_{1} \leq \lambda_{\max }\left(P_{z}\right)\|\mathbf{z}\|$, we have

$$
\begin{aligned}
\dot{V}_{1} & \leq-w_{o} L_{0}(1-\theta)\|\mathbf{z}\|^{2} \\
& \leq-L_{0} \frac{1-\theta}{\lambda_{\max }\left(P_{z}\right)} w_{o} V_{1} \leq-w_{o} L_{2} V_{1}, \forall \mathbf{z} \in R / \Omega_{\mathbf{z}}
\end{aligned}
$$

for some constant $L_{2}>0$, which leads to

$$
\begin{aligned}
\lambda_{\min }\left(P_{z}\right)\|\mathbf{z}\| & \leq V_{1} \leq e^{-w_{o} L_{2} t} V_{1}(\mathbf{z}(0)) \\
& \leq \lambda_{\max }\left(P_{z}\right)\|\mathbf{z}(0)\| e^{-w_{o} L_{2} t}, \forall \mathbf{z} \in R / \Omega_{\mathbf{z}}
\end{aligned}
$$

Hence, any trajectories starting outside $\Omega_{\mathbf{z}}$ will enter $\Omega_{\mathbf{z}}$ after $t=T\left(w_{o}\right)$ and $\lim _{w_{o} \rightarrow \infty} T\left(w_{o}\right)=0$. We have

$$
\|\tilde{\xi}\| \leq w_{o}^{\varrho}\|\mathbf{z}\| \leq O\left(w_{o}^{-1}\right)
$$


Equation (14) indicates that, for sufficiently small estimation error $\tilde{\xi}$, control signal $u$ is in the linear interval of $g_{w_{o}}(\cdot)$ :

$$
u=\frac{-K_{c} \bar{\xi}-\hat{\xi}_{\varrho, 2}}{b_{0}}
$$

Substituting (22) in to (18) leads to

$$
\begin{aligned}
\dot{\xi} & =A \xi+B\left(\phi_{1}-K_{c} \bar{\xi}-\frac{\left(\phi_{2}-b_{0}\right) K_{c} \bar{\xi}-\phi_{2} \hat{\xi}_{\varrho, 2}}{b_{0}}\right) \\
& =\left(A-B K_{c}\right) \xi+B\left(\begin{array}{ll}
\frac{\phi_{2}}{b_{0}} K_{c} & 1
\end{array}\right)\left(\begin{array}{c}
\mathcal{L} \tilde{\xi} \\
\tilde{\xi}_{\varrho, 2}
\end{array}\right) \\
& =\left(A-B K_{c}\right) \xi+B \Delta_{2}
\end{aligned}
$$

with

$$
\left\|\Delta_{2}\right\| \leq \iota_{2} w_{o}^{\varrho}\|\mathbf{z}\|
$$

where $\iota_{2}>0$. Repeating the previous work for system (20) now to system (23) would lead to the result that any trajectory $\xi(t)$ started in $\Omega_{\xi}$ will remain in this set for all $t>0$ and will enter $\left\{\|\xi\| \leq \frac{L_{1}}{w_{o}}\right\}$ within finite time $T\left(w_{o}\right)$. Since $y(0)=y^{*}(0)$ and $\dot{y}, \dot{y}^{*}$ are bounded, by the continuous dependence of the solution of state equation on initial states and parameters [18], we have

$$
\begin{array}{lll}
|y(t)-y *(t)|=O\left(T\left(w_{o}\right)\right), & & \forall t \in\left[0, T\left(w_{o}\right)\right] \\
|y(t)-y *(t)|=O\left(T\left(w_{o}\right)\right)+O\left(w_{o}^{-1}\right), & \forall t \in\left[T\left(w_{o}\right), \infty\right]
\end{array}
$$

Hence, we obtain

$$
\lim _{w_{o} \rightarrow \infty}\left|y(t)-y^{*}(t)\right|=0
$$

Because that

$$
|y(t)-0| \leq\left|y-y^{*}(t)\right|+\left|y^{*}(t)-0\right|
$$

For any given $\delta>0$, we can choose $w_{o}>0$ large enough such that

$$
\left|y-y^{*}(t)\right| \leq \frac{\delta}{2}, \quad \forall t \in[0, \infty]
$$

By the exponential stability of the target system, there exists $T_{1}\left(\delta, w_{c}\right)>0$ depending on $\delta$ and $w_{c}$ such that

$$
\left|y^{*}(t)-0\right| \leq \frac{\delta}{2}, \quad \forall t \in\left[T_{1}\left(\delta, w_{c}\right), \infty\right]
$$

Hence,

$$
|y(t)-0| \leq \delta, \quad \forall t \in\left[T_{1}\left(\delta, w_{c}\right), \infty\right]
$$

The proof is completed.

Remark 1: By Theorem 1, we have shown that, with a sufficiently large $w_{o}$, the steady state error would be rather small, and the output $y$ could be sufficiently close to target output $y^{*}$, which is exactly the performance recovery.

However, due to the continuously varying external disturbance $\mathbf{w}$, the asymptotic stability was not included; to obtain that, we need put restrictions on $\mathbf{w}$, and $f_{0}(\cdot)$.

Theorem 2: For closed-loop system $(17) \sim(19)$, if the following requirements are satisfied:

- $\mathbf{w}$ is constant, which indicates $\dot{\mathbf{w}}=0$.

- $f_{0}(\zeta, 0, \mathbf{w})=0$ has unique solution $\zeta_{s}(\mathbf{w})$, and $\zeta=\zeta_{s}$ is an exponentially stable equilibrium point of $\dot{\zeta}=$ $f_{0}(\zeta, 0, \mathbf{w})$.
- The system $\dot{\bar{\zeta}}=f_{0}\left(\bar{\zeta}+\zeta_{\mathbf{s}}, \xi, \mathbf{w}\right)$ is uniformly ISS [18] with respect to input $\xi$, where $\bar{\zeta}=\zeta-\zeta_{\mathrm{s}}$.

then, there exists $w_{o}^{*}>0$ such that for $w_{o}>w_{o}^{*}$, the output $y(t)$ satisfies

$$
\lim _{t \rightarrow \infty}|y(t)|=0
$$

Proof: First, rewrite the system as

$$
\begin{aligned}
\dot{\bar{\zeta}} & =f_{0}\left(\bar{\zeta}+\zeta_{\mathbf{s}}, 0, \mathbf{w}\right)+f_{0}\left(\bar{\zeta}+\zeta_{\mathbf{s}}, \xi, \mathbf{w}\right)-f_{0}\left(\bar{\zeta}+\zeta_{\mathbf{s}}, 0, \mathbf{w}\right) \\
& \stackrel{\text { def }}{=} f_{0}\left(\bar{\zeta}+\zeta_{\mathbf{s}}, 0, \mathbf{w}\right)+\Delta_{3}(\cdot) \\
\dot{\xi} & =\left(A-B K_{c}\right) \xi+\Delta_{2}(\cdot) \\
\dot{\mathbf{z}} & =w_{o} J z++w_{o} \Lambda_{1} B_{2 \varrho} C^{1} \mathbf{z}+B_{2 \varrho} \Delta_{1}(\cdot)
\end{aligned}
$$

According to the differentiability of $f_{0}(\cdot)$, we have

$$
\left\|\Delta_{3}\right\| \leq \iota_{1}\|\xi\|
$$

and some properties we obtained before

$$
\begin{aligned}
\left\|\Delta_{2}\right\| & \leq \iota_{2} w_{o}^{\varrho}\|\mathbf{z}\| \\
\left\|\Delta_{1}\right\| & \leq \iota_{3} w_{o}^{-\varrho}\|\bar{\zeta}\|+\iota_{4} w_{o}^{-\varrho}\|\xi\|+\iota_{5}\|\mathbf{z}\|
\end{aligned}
$$

for some positive constants $\iota_{i}, i=1, \cdots, 5$. According to Lyapunov converse theorem [18] and the ISS of internal dynamic $\dot{\bar{\zeta}}=f_{0}\left(\bar{\zeta}+\zeta_{\mathbf{s}}, \xi, \mathbf{w}\right)$, there exists positive definite function $V_{3}(\bar{\zeta})$ of system (25)satisfying

$$
\dot{V}_{3} \leq-\kappa_{1}\|\bar{\zeta}\|^{2}, \quad\left\|\frac{\partial V_{3}}{\partial \bar{\zeta}}\right\| \leq \kappa_{2}\|\bar{\zeta}\|
$$

and a positive definite function $V_{2}(\xi)$ whose derivative along $\xi(t, \xi(0))$ satisfies

$$
\dot{V}_{2} \leq-\kappa_{3}\|\xi\|^{2}, \quad\left\|\frac{\partial V_{2}}{\partial \xi}\right\| \leq \kappa_{4}\|\xi\|
$$

$\kappa_{i}, i=1,2,3,4$ are some positive constants. Let

$$
V=\kappa_{0} V_{2}+V_{3}+w_{o}^{2 \varrho} \mathbf{z}^{T} P_{z} \mathbf{z}
$$

with a positive constant $\kappa_{0}>0$. The time-derivative of $V$ along $(25) \sim(27)$ satisfies

$$
\begin{aligned}
\dot{V} & \leq-\kappa_{1}\|\bar{\zeta}\|^{2}-\kappa_{0} \kappa_{3}\|\xi\|^{2}-\left(w_{o}^{2 \varrho+1}-\iota_{5}\left\|P_{z}\right\| w_{o}^{2 \varrho}\right)\|\mathbf{z}\|^{2} \\
& +\kappa_{0} \kappa_{4}\|\mathbf{z}\|\|\xi\|+\kappa_{2}\|\xi\|\|\bar{\zeta}\|+w_{o}^{\varrho} \iota_{4}\left\|P_{z}\right\|\|z\|\|\xi\|+w_{o}^{\varrho} \iota_{3}\left\|P_{z}\right\|\|z\|\|\bar{\zeta}\| \\
& \leq-\left(\begin{array}{c}
\|\bar{\zeta}\| \\
\|\xi\| \\
\|\mathbf{z}\|
\end{array}\right)^{T}\left[\begin{array}{ccc}
\kappa_{1} & -\iota_{1} \kappa_{2} / 2 & O\left(w_{o}^{\varrho}\right) \\
-\iota_{1} \kappa_{2} / 2 & \kappa_{0} \kappa_{3} & O\left(w_{o}^{\varrho}\right) \\
O\left(w_{o}^{\varrho}\right) & O\left(w_{o}^{\varrho}\right) & w_{o}^{2} \varrho+1 \\
\end{array}\right]\left(\begin{array}{l}
\|\bar{\zeta}\| \\
\|\xi\| \\
\|\mathbf{z}\|
\end{array}\right)
\end{aligned}
$$

If $\kappa_{0}$ are chosen such that $\kappa_{0} \kappa_{1} \kappa_{3} \geq \iota_{1}^{2} \kappa_{2}^{2}$, the determinant will be

$$
w_{o}^{2 \varrho+1}\left|\begin{array}{cc}
\kappa_{1} & -\iota_{1} \kappa_{2} / 2 \\
-\iota_{1} \kappa_{2} / 2 & \kappa_{0} \kappa_{3}
\end{array}\right|-O\left(w_{o}^{\varrho}\right)
$$

Hence, by Sylvester criterion, there exists $w_{o}^{*}>0$ such that for every $w_{o}>w_{o}^{*}, \dot{V}$ satisfies

$$
\dot{V} \leq-\tau\left(\|\bar{\zeta}\|^{2}+\|\xi\|^{2}+\|\mathbf{z}\|^{2}\right)
$$

for some $\tau>0$. The proof is completed.

Remark 2: Theorem 2 is a complement to Theorem 1. It suggests that LPADRC has the "integral part" which is similar to PID controller. 


\section{Tuning procedure}

Considering the complexity of plants and variety of performance requirements, it seems that there is no universal criteria for selecting parameters. But we do have some useful principles for parameter-tuning. The first parameters to be set are $K_{i}, i=1, \cdots, \varrho$ because we need ensure that $J$ is a Hurwitz matrix; we give a simple law to assign all the eigenvalues of $J$ at $\lambda_{J}^{j}=-1,1 \leq j \leq 2 \varrho($ the same as LESO [19]):

$$
\begin{array}{ll}
k_{i, 1}=2 & , 1 \leq i \leq \varrho \\
k_{i, 2}=\frac{\varrho+1-i}{i} & , 1 \leq i \leq \varrho
\end{array}
$$

Next, we set $\beta_{i}, 1 \leq i \leq \varrho$ according to the rule:

$$
\beta_{i}=C_{\varrho}^{i-1}, 1 \leq i \leq \varrho
$$

where $C_{\varrho}^{i-1}$ denotes the binomial coefficient. The third to be set is $b_{0}$, whose effect has not been decided yet, but generally $b_{0}$ should satisfy

$$
b_{0} \geq 2 \max _{(\eta, \xi, \mathbf{w}) \in \Omega_{\zeta} \times \Omega_{\xi} \times \mathbb{D}_{\mathbf{w}}}\left|\phi_{2}(\zeta, \xi, \mathbf{w})\right|
$$

Recall that LPADRC can recover the performance of state-feedback control; accordingly, $w_{c}$ has little affect on stability if $w_{o}$ is large enough. Therefore, we shall first set the $w_{o}$, which decides the estimation-speed of LPESO. Then we choose an appropriate $w_{c}$ to satisfy requirements. Now, there is only $M$ to be set. There is an interplay between $w_{c}$ and $M$. If $M$ is too small for a fixed $w_{c}$, the system may oscillate. To summarize, the overall procedure of parameter-tuning is given in Figure 1:
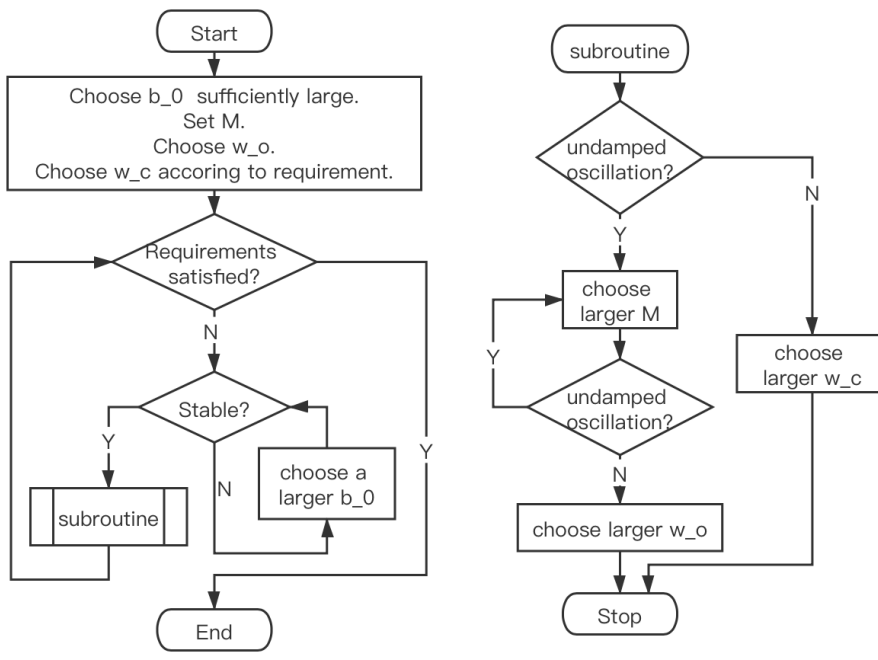

Fig. 1. The tuning procedure of LPADRC

Remark 3: There is an interplay between $w_{o}$ and $b_{0}$. Fix $w_{o}$, as $b_{0}$ increases, the decay speed of estimation-error will decrease, which indicates wore disturbance rejection. The increase of $b_{0}$ also affect the selection of $w_{c}$ by attenuating the control signal $u$.
TABLE I

NOMENCLATURE

\begin{tabular}{|ll|ll|}
\hline Symbol Denotation & Sub & Denotation \\
\hline$P$ & pressure(bar) & 0 & nominal value \\
$V$ & volume of chamber $\left(\mathrm{m}^{3}\right)$ & 1 & compressor inlet \\
$T$ & temperature $(\mathrm{K})$ & 2 & chamber inlet \\
$R$ & gas constant $(\mathrm{J} /(\mathrm{kg} \cdot \mathrm{K}))$ & 3 & turbine inlet \\
$\pi$ & pressure ratio & 4 & turbine outlet \\
$\sigma$ & pressure loss & $c$ & compressor \\
$L$ & torque $(\mathrm{N} \cdot \mathrm{m})$ & $t$ & turbine \\
$I$ & shaft inertia $\left(\mathrm{kg} \cdot \mathrm{m}^{2}\right)$ & $V$ & constant volume \\
$\gamma$ & adiabatic index & $f$ & fuel \\
$C$ & specific heat $(\mathrm{kJ} /(\mathrm{kg} \cdot \mathrm{K}))$ & $\mathrm{I}$ & inlet duct \\
$n$ & speed $(\mathrm{rpm})$ & $\mathrm{N}$ & nozzle \\
$\rho$ & density $\left(\mathrm{kg} / \mathrm{m}^{3}\right)$ & $\mathrm{P}$ & constant pressure \\
$\eta$ & efficiency & comb chamber \\
$v$ & gas flow rate $(\mathrm{kg} / \mathrm{s})$ & & electrical torque \\
$L H V$ & lower heating value of & \multicolumn{2}{|l}{} \\
\multicolumn{2}{|c|}{ fuel $(\mathrm{Jg})$} & & \multicolumn{2}{|l}{} \\
\hline
\end{tabular}

\section{Single Shaft Gas turbine CONTROL}

\section{A. Nonlinear model of SSGT}

Figure 2 shows the layout of a common SSGT consisting of inlet tube, compressor, combustion, turbine and nozzle. The compressor inhales air and increases its pressure. Then the high-pressure air entries the combustion chamber, where the fuel is added and then ignited to produce heat. The power generated by expanding hot and highpressure gas through the turbine will be exhausted by both compressor and electric load.

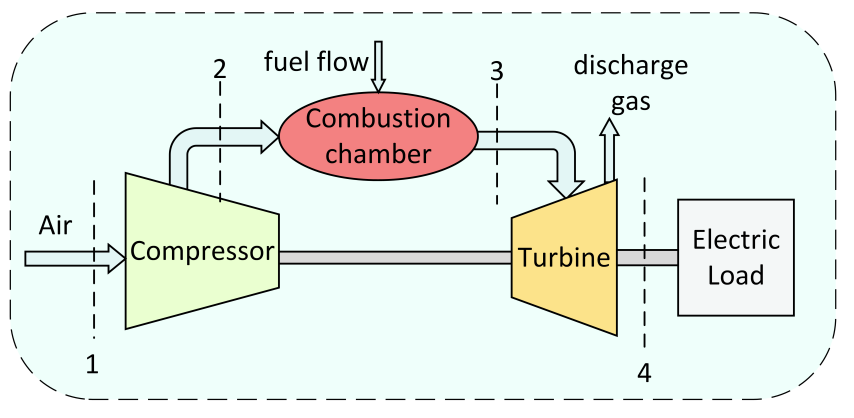

Fig. 2. Layout of a common single shaft gas turbine used for electric generation consisting of a compressor, a combustion chamber and a turbine.

Generally, when gas turbine works in the steady state, the power generated by the turbine is equal to the sum of power absorbed by the compressor and exhausted by the electric load. If power balance is disturbed by change in electric load or ambient condition, there is a dynamic process in the chamber. Consequently, it will lead to pressure and temperature change.

According to [5], three differential equations (31), which derived from the conversation law of mass and momentum, 
represents the original SSGT model.

$$
\begin{aligned}
\frac{\mathrm{d} P_{3}}{\mathrm{~d} t}= & \frac{R T_{3}}{V}\left(v_{c}+v_{f}-v_{t}\right) \\
\frac{\mathrm{d} T_{3}}{\mathrm{~d} t}= & \left\{v_{c} C_{P} T_{1}\left[\left(\pi_{c}^{\frac{\gamma-1}{\gamma}}-1\right) / \eta_{c}+1\right]-v_{t} C_{P} T_{3}+v_{f} L H V\right. \\
& \left.-C_{V} T_{3}\left(v_{c}-v_{t}\right)\right\} /\left(\rho_{2} V C_{V}\right) \\
\frac{\mathrm{d} n}{\mathrm{~d} t}= & \frac{900}{\pi^{2} I n}\left\{v_{t} C_{P} T_{3}\left(1-\pi_{t}^{\frac{1-\gamma}{\gamma}}\right) \eta_{t}\right. \\
& \left.-v_{c} C_{P} T_{1}\left(\pi_{c}^{\frac{\gamma-1}{\gamma}}-1\right) / \eta_{c}-\frac{2 \pi}{60} n L_{e}\right\}
\end{aligned}
$$

where $\pi_{c}$ and $\pi_{t}$ are the pressure ratio given as

$$
\pi_{c}=\frac{P_{2}}{P_{1}}=\frac{P_{3}}{\sigma_{c o m b} P_{1}}, \quad \pi_{t}=\frac{P_{3}}{P_{4}}=\frac{P_{3} \sigma_{I} \sigma_{N}}{P_{1}}
$$

The gas flow rate $v_{c}$ and $v_{t}$ are computed as the functions of pressure ratio and rotational speed, and we need to derive them from the experimental data. Figure 3 shows a typical compressor characteristic map derived from experimental data, which consists of two sets of curves. The first set describes the relation between pressure ratio $\pi_{c}$ and non-dimensional air flow rate $\frac{v_{c} \sqrt{T_{1}}}{P_{1}}$ under the same speed. The second set describes the relation between efficiency $\eta_{c}$ and $\frac{v_{c} \sqrt{T_{1}}}{P_{1}}$ under same speed. Similarly, the turbine characteristic map describes same relations for turbine. With these maps/data, gas flow rate of compressor and turbine can be represented approximately by functions:

$$
\begin{aligned}
& v_{c}=g_{1}\left(\frac{n}{\sqrt{T_{1}}}, \pi_{c}\right) \cdot \frac{P_{1}}{\sqrt{T_{1}}}=f_{1}\left(n, P_{3}, P_{1}, T_{1}\right) \\
& v_{t}=g_{2}\left(\frac{n}{\sqrt{T_{3}}}, \pi_{t}\right) \cdot \frac{P_{3}}{\sqrt{T_{3}}}=f_{2}\left(n, P_{3}, P_{1}, T_{3}\right)
\end{aligned}
$$

where $g_{1}(\cdot)$ and $g_{2}(\cdot)$ are continuously differentiable functions. It needs to be noticed that $\eta_{c}$ and $\eta_{t}$ varies slowly and slightly during the transition process compared to the gas flow rate. For simplicity, we treat them as constants [6].

Let $x=\left(\begin{array}{lll}x_{1} & x_{2} & x_{3}\end{array}\right)^{T}=\left(\begin{array}{lll}P_{3} & T_{3} & n\end{array}\right)^{T}, u=v_{f}$, $y(t)=n$. Substituting (33) into (31) leads to the statespace form:

$$
\begin{aligned}
& \dot{x}=\left(\begin{array}{l}
\psi_{1}(x, \mathbf{w}) \\
\psi_{2}(x, \mathbf{w}) \\
\psi_{3}(x, \mathbf{w})
\end{array}\right)+\left(\begin{array}{c}
b_{1} x_{2} \\
b_{2} \\
0
\end{array}\right) u \\
& y=\left(\begin{array}{lll}
0 & 0 & 1
\end{array}\right) x
\end{aligned}
$$

where $\mathbf{w}=\left(\begin{array}{lll}P_{1} & T_{1} & L_{e}\end{array}\right)^{T}$ represents the external disturbance and $b_{1}, b_{2}$ are positive constants given by :

$$
b_{1}=\frac{R}{V} \quad b_{2}=\frac{L H V}{\rho_{2} V c_{V}}
$$

$\psi_{i}(x, w), i=1,2,3$ are nonlinear continuously differen- tiable functions in the form of:

$$
\begin{aligned}
\psi_{1}(x, w)= & \frac{R}{V} x_{2}\left[f_{1}(x, w)-f_{2}(x, w)\right] \\
\psi_{2}(x, w)= & \frac{1}{\rho_{2} V c_{V}}\left\{f_{1}(x, w) c_{P} T_{1}\left[1-\frac{1}{\eta_{c}}+\frac{\left(\frac{x_{1}}{P_{1} \sigma_{c o m b}}\right)^{\frac{\gamma-1}{\gamma}}}{\eta_{c}}\right]\right. \\
& \left.-f_{2}(x, w) c_{P} x_{2}-c_{V}\left(f_{1}(x, w)-f_{2}(x, w)\right)\right\} \\
\psi_{3}(x, w)= & \frac{900}{\pi^{2} I n}\left\{f_{2}(x, w) c_{P} x_{2}\left[1-\left(\frac{x_{1} \sigma_{I} \sigma_{N}}{P_{1}}\right)^{\frac{1-\gamma}{\gamma}}\right] \eta_{t}\right. \\
- & \left.f_{1}(x, w) c_{P} T_{1}\left[\left(\frac{x_{1}}{P_{1} \sigma_{c o m b}}\right)^{\frac{\gamma-1}{\gamma}}-1\right] / \eta_{c}-\frac{2 \pi}{60} x_{3} L_{e}\right\}
\end{aligned}
$$

where $f_{1}(\cdot)$ and $f_{2}(\cdot)$ are aforementioned.

It can be seen that the exact mathematical model is unavailable due to some unavoidable approximation and unknown external disturbance. By Lemma 1, we can easily verify that the transient SSGT model (34) has relative degree $\varrho=2$. Then, a 2-nd order LPADRC will be designed.

\section{B. LPADRC for SSGT and noise attenuation}

To investigate the influence of measurement noise on control signal $u$, we first linearize a specific SSGT model at steady state and then derive the transfer function $\frac{u(s)}{\nu(s)}$.

The SSGT data is obtained form National Aeronautics and Space Administration (NASA) in its open-source project. The first step would be interpolating the gas flow function $v_{c}$ and $v_{t}$ based on the characteristic maps; we take a polynomial function (35):

$$
f(n, \pi)=\text { cont }_{1}+\text { cont }_{2} * \pi+\text { cont }_{3} * n+\text { cont }_{4} * \pi n
$$

where cont $_{i}, i=1, \cdots, 4$ are constants. Recall that $x_{1}=$ $P_{1}, x_{2}=T_{3}, x_{3}=n$.

The interpolation results are listed as below:

$v_{c}=-19.8+0.0003632 x_{1} x_{3}-3.09 x_{1}+0.005 x_{3}$

$v_{t}=101 x_{1} x_{2}^{-\frac{1}{2}}-6.04 \times 10^{-6} x_{1} x_{3}-0.073 x_{1}^{\frac{3}{2}} x_{2}^{-\frac{1}{2}}+3.6 \times 10^{-5} x_{1}^{2} x_{3}$

The other parameters of SSGT at the steady state are given in Table II.

TABLE II

THE VALUES OF PARAMETERS OF A SPECIFIC SINGLE SHAFT GAS TURBINE

\begin{tabular}{|l|l|l|l|l|l|}
\hline symbol & value & symbol & value & symbol & value \\
\hline $\mathrm{n}(\mathrm{rpm})$ & 10000 & $T_{0}$ & 288.15 & $P_{0}$ & 1 \\
$T_{3}$ & 1710 & $P_{3}$ & 19.4 & $c_{P}$ & 1004 \\
$c_{V}$ & 717 & $L_{e}$ & 0 & $I$ & 30 \\
$\gamma$ & 1.4 & $R$ & 287 & $L H V$ & $4.08 \times 10^{7}$ \\
$\sigma_{\text {comb }}$ & 0.95 & $\sigma_{I}$ & 0.98 & $\sigma_{N}$ & 0.99 \\
$\eta_{c}$ & 0.8043 & $\eta_{t}$ & 0.9013 & & \\
\hline
\end{tabular}

By linearization and shifting equilibrium point $x=$ $(19.4,1710,10000)$ to $(0,0,0)$, the linear model is devel- 

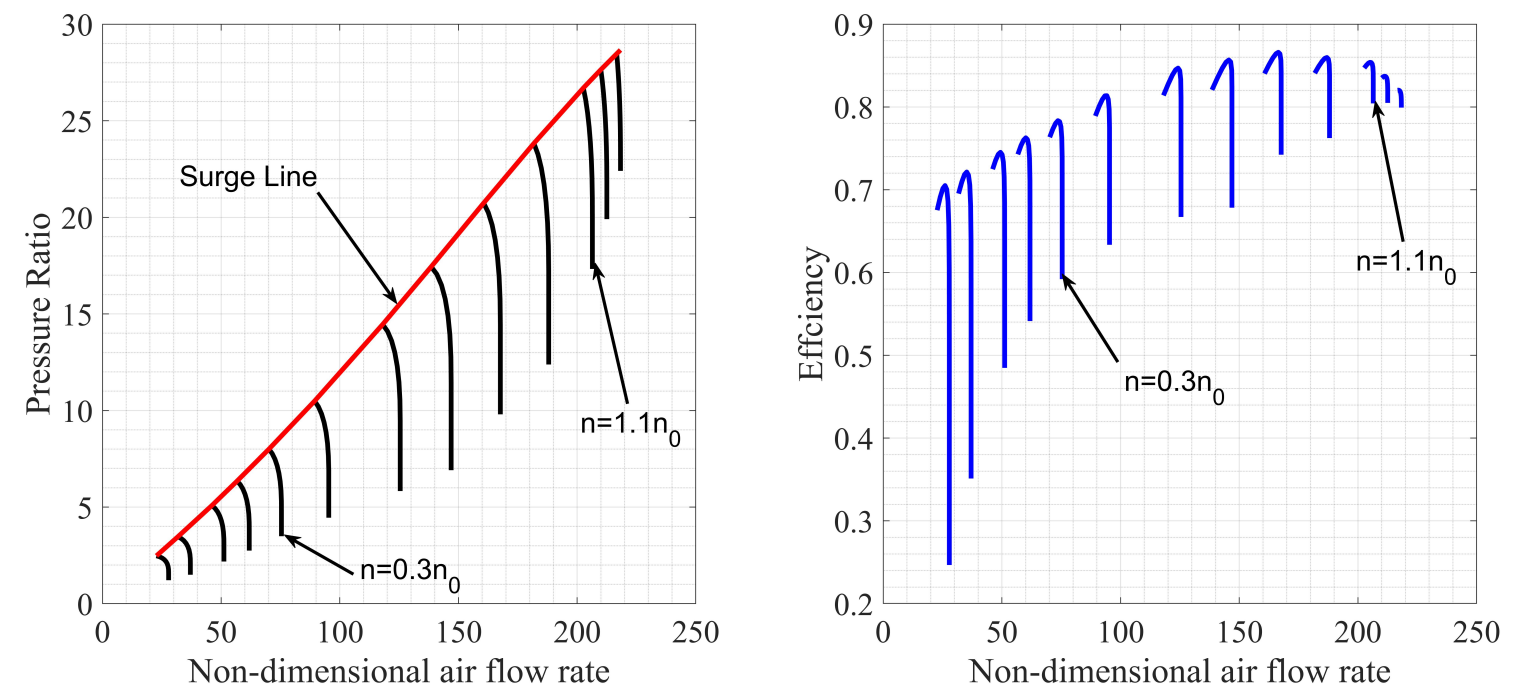

Fig. 3. Compressor characteristic map: The left figure is the map about non-dimensional air flow rate and Pressure ratio. The right figure is the map about non-dimensional air flow rate and efficiency. The symbol $\boldsymbol{n}_{\mathbf{0}}$ denotes the nominal rotational speed.

oped :

$\dot{x}=\left[\begin{array}{ccc}-1466 & -5.76 & 0.0055 \\ -59000 & -328.4 & 16.05 \\ -4400 & -22 & 2.35\end{array}\right] x+\left[\begin{array}{c}98 \\ 95000 \\ 0\end{array}\right] u=A x+B u$

The LPESO and the feedback law are given as:

$$
\begin{gathered}
{\left[\begin{array}{c}
\dot{\hat{\xi}}_{1,1} \\
\dot{\hat{\xi}}_{1,2} \\
\dot{\hat{\xi}}_{2,1} \\
\dot{\hat{\xi}}_{2,2}
\end{array}\right]=} \\
+\left[\begin{array}{cccc}
-2 w_{o} & 1 & 0 & 0 \\
-2 w_{o}^{2} & 0 & 0 & 1 \\
0 & 2 w_{o} & -2 w_{o} & 1 \\
0 & \frac{1}{2} w_{o}^{2} & -\frac{1}{2} w_{o}^{2} & 0
\end{array}\right] \hat{\xi}+\left[\begin{array}{c}
0 \\
b_{0} \\
b_{0} \\
0
\end{array}\right] u \\
+\left[\begin{array}{c}
2 w_{o} \\
2 w_{o}^{2} \\
0 \\
0
\end{array}\right]\left(x_{3}+\nu(t)\right) \\
\dot{\hat{\xi}}=F \hat{\xi}+B_{e} u+B_{\nu} y+B_{\nu} \nu(t) \\
u=\frac{1}{b_{0}}\left[\begin{array}{ccc}
-w_{c}^{2} & \left.0-2 w_{c}-1\right] \hat{\xi}=C_{c} \hat{\xi}
\end{array}\right.
\end{gathered}
$$

where $\nu(t)$ represents the bounded high-frequency measurement noise. Notice that, in steady state, control signal $u$ satisfies $|u|<M$. Hence, here we drop the analogous saturation function in (37). Now, the whole closed-loop system can be represented as

$$
\begin{aligned}
{\left[\begin{array}{c}
\dot{x} \\
\dot{\hat{\xi}}
\end{array}\right] } & =\left[\begin{array}{cc}
A & B * C_{c} \\
B_{\nu} *\left[\begin{array}{lll}
0 & 0 & 1
\end{array}\right] & F+B_{e} * C_{c}
\end{array}\right]\left[\begin{array}{c}
x \\
\hat{\xi}
\end{array}\right]+\left[\begin{array}{c}
0_{3 \times 1} \\
B_{\nu}
\end{array}\right] \nu(t) \\
& =\mathcal{A}\left[\begin{array}{l}
x \\
\hat{\xi}
\end{array}\right]+\mathcal{B} \nu(t) \\
u & =\left[\begin{array}{ll}
0_{1 \times 3} & C_{c}
\end{array}\right]\left[\begin{array}{l}
x \\
\hat{\xi}
\end{array}\right]=\mathcal{C}\left[\begin{array}{c}
x \\
\hat{\xi}
\end{array}\right]
\end{aligned}
$$

Then, the transfer function $\frac{u(s)}{\nu(s)}$ will be

$$
T_{L P E S O}=\frac{u(s)}{\nu(s)}=\mathcal{C}(s I-\mathcal{A})^{-1} \mathcal{B}
$$

For comparison, a LESO designed for this SSGT is given:

$$
\dot{z}=\left[\begin{array}{ccc}
-3 w_{o} & 1 & 0 \\
-3 w_{o}^{2} & 0 & 1 \\
-w_{o}^{3} & 0 & 0
\end{array}\right] z+\left[\begin{array}{c}
3 w_{o} \\
3 w_{o}^{2} \\
w_{o}^{3}
\end{array}\right]\left(x_{3}+\nu(t)\right)
$$

and the feedback law is

$$
u=\frac{1}{b_{0}}\left[\begin{array}{lll}
-w_{c}^{2} & -2 w_{c} & -1
\end{array}\right] z
$$

By repeating the same procedure to LESO, we obtain the transfer function $T_{L E S O}=\frac{u(s)}{\nu(s)}$ for the LESO based strategy.

Now, with the same $w_{o}, w_{c}, b_{0}$ for both two control schemes, a Bode-magnitude plot is given to visually compare the frequency noise attenuation feature of LPADRC and LADRC. It is seen from Figure 4 that, the amplification of high frequency noise $\nu(t)$ of LPADRC is much lower than LADRC.

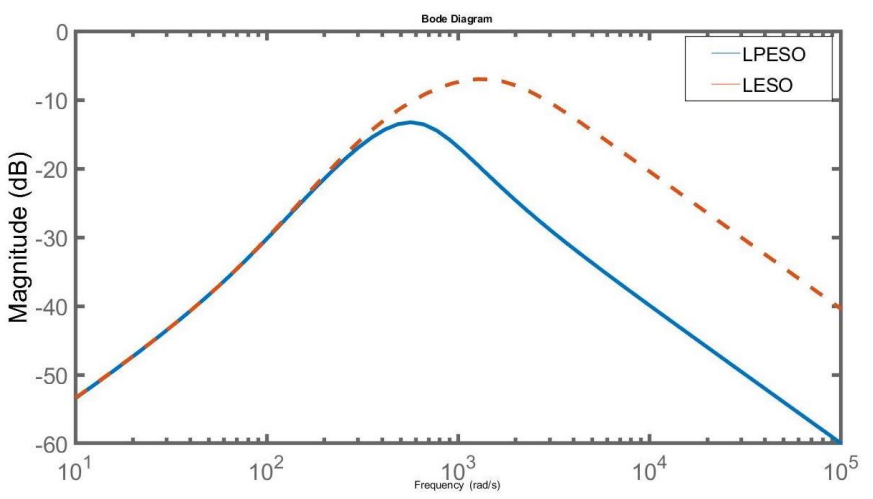

Fig. 4. The Bode-magnitude plot of $\frac{\boldsymbol{u}(\boldsymbol{s})}{\boldsymbol{\nu}(\boldsymbol{s})}$ of LPADRC and LADRC, respectively. Both scheme has the same $\boldsymbol{w}_{\boldsymbol{o}}, \boldsymbol{w}_{\boldsymbol{c}}, \boldsymbol{b}_{\mathbf{0}}$. It is seen clearly that within the high-frequency band, LPESO has much smaller noiseamplification than LESO. 


\section{Numerical simulations for transient process}

To illustrate the superiority and effectiveness of the proposed scheme, simulations are carried out using the "T-MATS" toolbox developed by NASA in MATLAB/Simulink. We compare the performance of PID, 2ndorder ADRC, 2nd-order LPADRC (the proposed scheme). Figure 5 shows the layout of the closed-loop system.

The specifications of the SSGT and parameters of controllers are given in Table III and Table IV. The sample time is taken as $T_{s}=0.0001$. High-Frequency noise is generated by the band-limited white noise block together with a high-pass filter $G(s)=\frac{s}{s+500}$. We simulate experiments of step change in load, and gradual change in altitude, respectively.

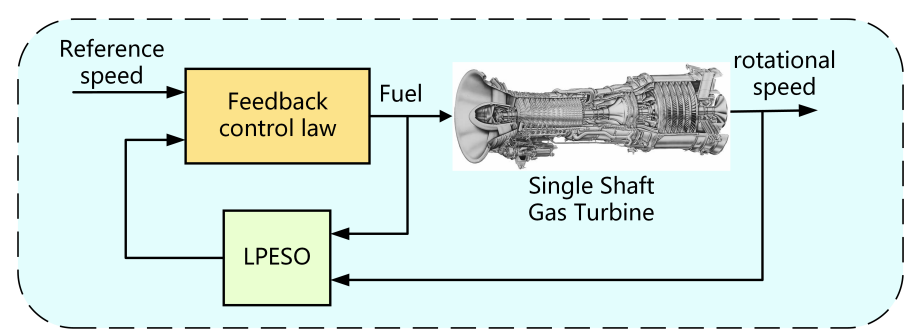

Fig. 5. Schematic diagram of the gas turbine fuel flow rate control system. The SSGT graphic is from Neilson [20]

In practice, the main restriction is the trade-off between disturbance rejection and control signal noisy level. It is well known that, increasing the gain of LADRC would leads to higher noisy level of control signal [19]; too noisy signal will damage actuators. Here are our criteria for comparison: 1) better disturbance rejection with the same noisy $u 2$ ) less noisy $u$ with the same disturbance rejection performance.

TABLE III

SPECIFICATIONS OF THE GAS TURBINE USED FOR THIS SIMULATION.

\begin{tabular}{|l|l|}
\hline Description & Specification \\
\hline Type & single shaft \\
Nominal speed & $10000($ rpm $)$ \\
Nominal power & $100($ horsepower $)$ \\
Compression ratio & 20 \\
\hline
\end{tabular}

TABLE IV

PARAMETERS OF PID, ADRC, LPADRC.

\begin{tabular}{|l|l|}
\hline Controller & Parameters \\
\hline PI & $P=0.01, I=0.05$ \\
LADRC & $w_{o}=50, w_{c}=20, b_{0}=50000, M=3$ \\
LPADRC & $w_{o}=100, w_{c}=20, b_{0}=50000, M=3$ \\
\hline
\end{tabular}

1) Simulation for altitude change: The altitude of environment affects the ambient temperature $T_{0}$ and ambient pressure $P_{0}$. Consequently, change in altitude will trigger a transient process. We need assure an effective control when it suffers an gradual altitude change:

$$
\text { Altitude }(f t)= \begin{cases}0 & t \leq 4 s \\ 5000(t-4) & 4 s \leq t \leq 6 s \\ 10000 & 6 s \leq t\end{cases}
$$

According to the criteria we set before, Figure 6 shows that proposed scheme achieved better disturbance rejection with the same noisy level of control signal $u$.

2) Simulation for step load change: The changes in electric load are frequently encountered in practice, which could be caused by sudden transmission line fault or decrease in electricity consumption, etc. The load change with time:

$$
\operatorname{Load}(h p)= \begin{cases}100 & t \leq 5 s \\ 50 & 5 s<t\end{cases}
$$

Figure 7 indicates the same conclusion as Figure 6 .

\section{CONCLUSIONS}

This study set out to provide an alternative to LADRC by the Low Power Active Disturbance Rejection Control. The performance recovery of LPADRC has been proved via Lyapunov method, which makes it the second variant of ADRC possessing this property. Then, to facilitate the engineering implementation, we provided a simple observer parameter-configuration method and then the whole parameter-tuning procedure of the controller; the observer parameter-configuration method could also be adopted to other standard low power high gain observer. Furthermore, the gap between ADRC design and SSGT model caused by unmatched disturbances has been bridged by introducing the concept of relative degree. Finally, the effectiveness and superiority of the proposed scheme were verified by utilizing NASA's TMATS toolbox with a high-fidelity gas turbine model. The simulation results, in both graphical and numerical form, showed that LPADRC improved the disturbance rejection performance without degenerating the control signal when the measured output suffers from high-frequency noise.

However, the peaking-phenomenon problem, which is also a classic problem of high gain observer, has not been solved. We adopted the conventional method by restricting the magnitude of the control signal. In the future, we will focus on solving that problem. Besides that, the merit "low power" is not distinct for low-order system; so, it would be a quite interesting topic to apply LPADRC to some high-order systems.

\section{RefERENCES}

[1] J. Han, "From PID to active disturbance rejection control," IEEE Transactions on Industrial Electronics, vol. 56, no. 3, pp. 900-906, 2009.

[2] T. He, Z. Wu, D. Li, and J. Wang, "A Tuning Method of Active Disturbance Rejection Control for a Class of High-order Processes," IEEE Transactions on Industrial Electronics, pp. $1-11,2019$.

[3] W. Xue, W. Bai, S. Yang, K. Song, Y. Huang, and H. Xie, "ADRC with Adaptive Extended State Observer and its Application to Air-Fuel Ratio Control in Gasoline Engines," IEEE Transactions on Industrial Electronics, vol. 62, no. 9, pp. 5847$5857,2015$.

[4] H. Sira-Ramírez, J. Linares-Flores, C. García-Rodríguez, and M. A. Contreras-Ordaz, "On the control of the permanent magnet synchronous motor: An active disturbance rejection control approach," IEEE Transactions on Control Systems Technology, vol. 22, no. 5, pp. 2056-2063, 2014.

[5] A.M.Y.Razak, Industrial Gas Turbines:Performance and operability. Cambridge: Woodhead Publishing, 2007. 

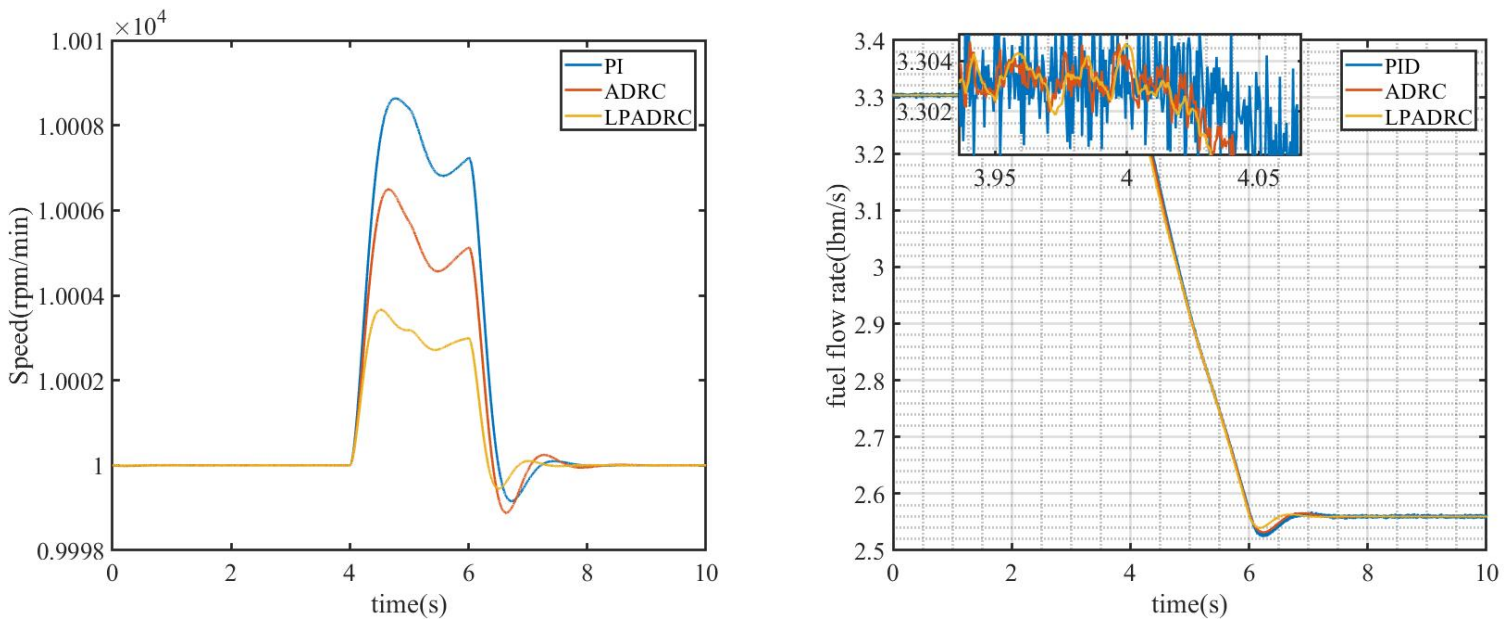

Fig. 6. Transient process of output $\boldsymbol{y}$ and control signal $\boldsymbol{u}$ when suffering an altitude change for PI, LADRC and LPADRC based control strategy. When suffers from a gradual altitude change disturbance, in all three control strategy, LPADRC based strategy has the smallest overshoot and the best undisturbed control signal. The integral of steady state error Int $=\int_{0}^{\mathbf{3 . 5}}\left|\boldsymbol{u}-\boldsymbol{u}_{\text {average }}\right| \boldsymbol{d t}$ are: Int $_{P I}=0.00470$, Int $_{L A D R C}=0.0191$, Int $_{L P A D R C}=0.00175$
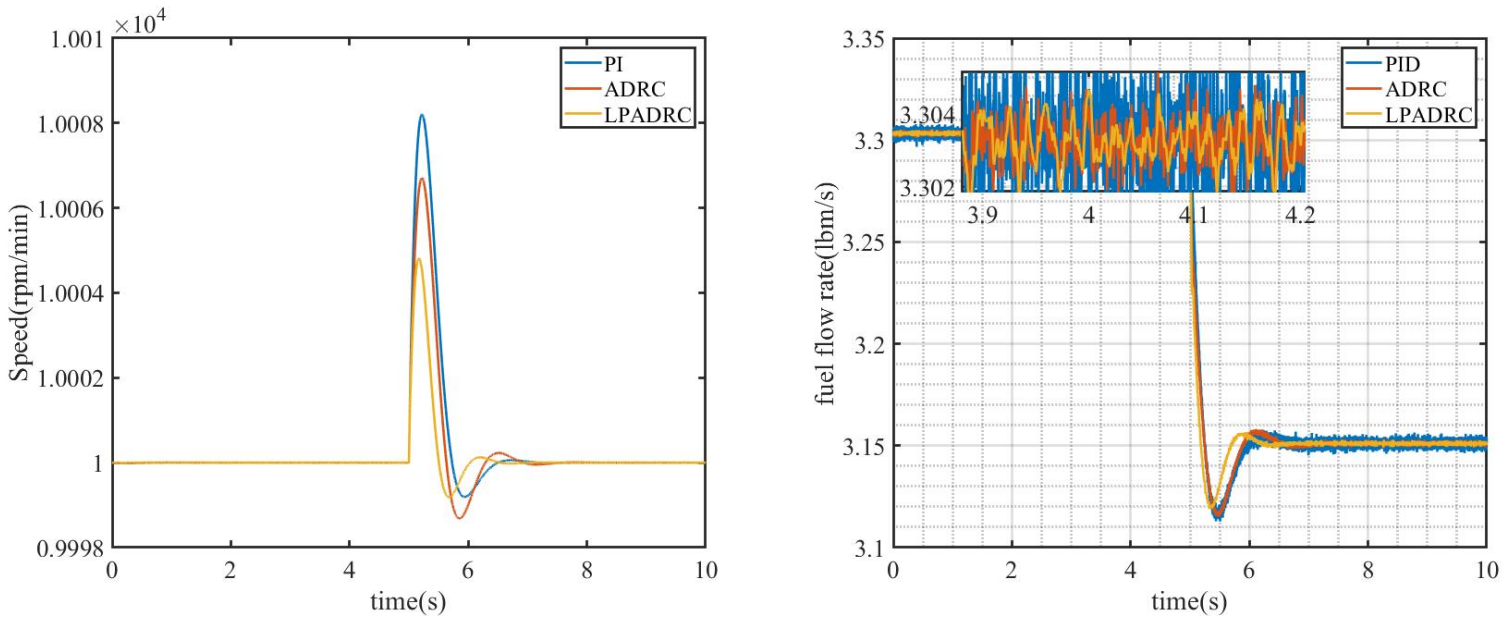

Fig. 7. Transient process of output $\boldsymbol{y}$ and control signal $\boldsymbol{u}$ when suffering an step load change for PI, LADRC and LPADRC based control scheme. This result leads to the same conclusion with the first simulation. The integral of steady state error Int $=\int_{\mathbf{0}}^{4.5}\left|\boldsymbol{u}-\boldsymbol{u}_{\boldsymbol{a}} \boldsymbol{v e r a g e}\right| \boldsymbol{d t}$ are: Int $_{P I}=0.00561$, Int $_{L A D R C}=0.0229$, Int $_{L P A D R C}=0.00212$

[6] H. I. Saravanamuttoo, G. F. C. Rogers, and H. Cohen, Gas turbine theory. Pearson Education, 2009.

[7] F. Gu, I. Yesilyurt, Y. Li, G. Harris, and A. Ball, "An investigation of the effects of measurement noise in the use of instantaneous angular speed for machine diagnosis," Mechanical Systems and Signal Processing, vol. 20, no. 6, pp. 1444-1460, 2006.

[8] A. P. Wiese, M. J. Blom, C. Manzie, M. J. Brear, and A. Kitchener, "Model reduction and MIMO model predictive control of gas turbine systems," Control Engineering Practice, vol. 45, pp. 194-206, 2015.

[9] B.-Z. Guo and Z.-L. Zhao, Active disturbance rejection control for nonlinear systems: An introduction. John Wiley \& Sons, 2016.

[10] L. B. Freidovich and H. K. Khalil, "Performance recovery of feedback-linearization-based designs," IEEE Transactions on Automatic Control, vol. 53, no. 10, pp. 2324-2334, 2008.

[11] D. Astolfi and G. Casadei, "Stabilization of nonlinear systems in presence of filtered output via extended high-gain observers," Automatica, vol. 110, p. 108594, 2019.

[12] X. Li and H. Xia, "A New Extended State Observer with Low Sensitivity to High Frequency Noise and Low Gain Power," 21th
IFAC World Congress, in press, 2020.

[13] D. Astolfi and L. Marconi, "A high-gain nonlinear observer with limited gain power," IEEE Transactions on Automatic Control, vol. 60, no. 11, pp. 3059-3064, 2015.

[14] H. K. Khalil, High-gain observers in nonlinear feedback control. SIAM, 2017.

[15] H. K. Khalil and S. Priess, "Analysis of the Use of LowPass Filters with High-Gain Observers," IFAC-PapersOnLine, vol. 49, no. 18, pp. 488-492, 2016.

[16] Z. L. Zhao and B. Z. Guo, "A nonlinear extended state observer based on fractional power functions," Automatica, vol. 81, no. April, pp. 286-296, 2017.

[17] A. Isidori, Nonlinear control systems. Springer Science \& Business Media, 2013.

[18] H. Khalil, Nonlinear Systems, ser. Pearson Education. Prentice Hall, 2002.

[19] Z. Gao, "Scaling and bandwidth-parameterization based controller tuning," Proceedings of the 2003 American Control Conference, 2003., vol. 6, pp. 4989-4996, 2003.

[20] C. E. Neilson, "LM2500 gas turbine modifications for biomass fuel operation," Biomass and Bioenergy, vol. 15, no. 3, pp. 269$273,1998$. 